\title{
INTELLECTUAL DATA ANALYSIS IN AIRCRAFT DESIGN
}

\author{
V.A. Komarov ${ }^{1}$, S.A. Piyavskiy ${ }^{2}$ \\ ${ }^{1}$ Samara National Research University, Samara, Russia \\ ${ }^{2}$ Samara State Architectural University, Samara, Russia
}

\begin{abstract}
This article considers the use of confidence judgments method by decision-makers to analyze the information contained in large databases. The comparative analysis of passenger aircrafts shows that it allows flexibly and objectively allocating the most relevant information from the data array.
\end{abstract}

Keywords: data analysis, aircraft design, large databases, decision-makers, confident judgments.

Citation: Komarov VA, Piyavskiy SA. Intellectual data analysis in aircraft design. CEUR Workshop Proceedings, 2016; 1638: 873-881. DOI: 10.18287/1613-0073-2016-1638-873-881

\section{Introduction}

The emergence of large corporate databases opens up new prospects in the field of aircraft design, as well as in other subject fields of project activity. It becomes possible to estimate comprehensively, over a large number of characteristics, both quantitative and qualitative, the efficiency of different variants of design decisions against a background of a huge amount of analogues. It is also important to keep in mind that the project activity has largely heuristic nature, based on a combination of objective quantitative analysis within intuitive the designers' ideas, arising impulses of which may the expand and transfer the attention focus, and even change the design paradigm itself. The mechanism of using large databases for the design of complex, multifunction objects such as aircraft should be oriented towards these features.

In our opinion, it is advisable to use some advanced methods of complex decision theory, such as multi-criteria optimization, during the formation of such mechanism. One of the main advantages of these methods is that they provide an adequate active role of decision-makers along with the use of axiomatic approach to the information analysis. For all the variety of decision-making methods [1,2], a decision-making method under irremovable uncertainly [3,4] and confident judgments method (CJM) are the most efficient methods from this points of view. The article is aimed to demonstrate opportunities offered by the application of these techniques during intellectual data analysis. The considered examples are given with great simplifications. 
Each object, denoted by y in the following, is described by a set of data which is useful to divide into two groups. In the first group it is advisable to include the data which determine how an object is arranged and which can be changed by decisionmakers. In terms of non-linear mathematical programming, it is usually called design variables. The data, which include the object's behavior characteristics or properties, should be contained in the second group. These data are of interest for products' customers, as a rule, in the form of maximum and minimum values. Further, we will denote them as a particular optimal criterion $\mathrm{f} \mathrm{i} \mathrm{(y).} \mathrm{In} \mathrm{most} \mathrm{cases,} \mathrm{the} \mathrm{particular} \mathrm{effi-}$ ciency criteria for complex technical objects are contradictory, which generates the well-known problem of multi-disciplinary optimization.

In aviation, for example, two important characteristics are in such conflict: the aircraft weight and aerodynamic efficiency. Increasing the aerodynamic quality is achieved by the wing lengthening, but it increases its weight $[7,8]$. Introducing new nondimensional load-carrying coefficient of structural perfection into consideration allows to carry out the optimization of aircraft appearance taking into account both weight and aerodynamic efficiency [9]. However, the design of new aircraft and, particularly, the development of technical specification for its creation, needs analysis and taking into account a variety of parameters, which can allow to predict the success of a new project by the consumer.

\section{Rating Estimation Method}

Let us consider a corporate database for the aircrafts as a set $Y$ of objects $y \in Y$, which are characterized by $m$-dimensional vectors $f(y)=\left\{f^{1}(y), f^{2}(y), \ldots, f^{m}(y)\right\}, y \in Y$. The components of these vectors are separate efficiency characteristics of the object, which are of interest from the viewpoint of decision-makers. For example, a passenger aircraft has the following characteristics considered from an operational point of view:

- Cruise speed, $\mathrm{km} / \mathrm{h}$

- Number of passengers, pers

- Flight range, km

- Service ceiling, $\mathrm{m}$

- Runway length, m

- Minimum price in passenger version, million USD

- Maximum price in passenger version, million USD

- Starting year of manufacturing

- Number of built aircrafts

- Engine power, kgf

- Fuel capacity, 1

Traditionally, the simplest way to analyze this data array is to sort by the values of any characteristics $f^{j}, j=1, \ldots, m$. It allows to define the locations according to solution variants for this characteristic among analogues. However, since the solution 
efficiency, in general, is determined mainly by its characteristics, the analytical value of sorting is not high.

More powerful tool for intellectual analysis is the allocation of the total array of objects which are Pareto efficient. The object is considered as Pareto efficient if there do not exist at least one dominant object on the entire considered set. It means that any object according to the characteristics not worse, and at least one - better. Thus, among the aircrafts, whose characteristics are given in Table 1 (data are taken from [7] and other sources, partly modeled and have purely methodological nature), Pareto efficient are Boeing 737-200, Boeing 737-400, Boeing 737-500 and Boeing 737-200 Advance is not efficient as it is dominated by Boeing 737-500.

Table 1. Some characteristics of Boeing's aircrafts

\begin{tabular}{|c|c|c|c|c|c|}
\hline Aircrafts & $\begin{array}{r}\text { Flight } \\
\text { range, } \mathrm{km}\end{array}$ & $\begin{array}{l}\text { Ceil- } \\
\text { ing, } m\end{array}$ & $\begin{array}{l}\text { Run } \\
\text { way } \\
\text { length, } \\
\text { m }\end{array}$ & $\begin{array}{l}\text { En- } \\
\text { gine } \\
\text { thrust, } \\
\text { kgf }\end{array}$ & $\begin{array}{r}\text { Fuel } \\
\text { capacity, } \\
1\end{array}$ \\
\hline $\begin{array}{l}\text { Boeing 737-200 Ad- } \\
\text { vance }\end{array}$ & 2960 & $\begin{array}{l}1067 \\
0\end{array}$ & $\begin{array}{l}183 \\
0\end{array}$ & $\begin{array}{l}1578 \\
0\end{array}$ & $\begin{array}{l}1953 \\
5\end{array}$ \\
\hline Boeing 737-300 & 4670 & $\begin{array}{l}1020 \\
0\end{array}$ & $\begin{array}{l}194 \\
0\end{array}$ & $\begin{array}{l}1994 \\
0\end{array}$ & $\begin{array}{l}2010 \\
5\end{array}$ \\
\hline Boeing 737-400 & 3870 & $\begin{array}{l}1130 \\
0\end{array}$ & $\begin{array}{l}192 \\
0\end{array}$ & $\begin{array}{l}2134 \\
0\end{array}$ & $\begin{array}{l}2382 \\
5\end{array}$ \\
\hline Boeing 737-500 & 5550 & $\begin{array}{l}1130 \\
0 \\
\end{array}$ & $\begin{array}{l}153 \\
0 \\
\end{array}$ & $\begin{array}{l}1816 \\
0 \\
\end{array}$ & $\begin{array}{l}2010 \\
5 \\
\end{array}$ \\
\hline
\end{tabular}

The rigorous formulation of Pareto efficient object is the following:

$$
\left.\neg \exists \widehat{y} \in Y:\left(f^{j}(\widehat{y}) \leq f^{j}(\bar{y}) j=1, \ldots, m\right) \wedge\left(\exists j \in\{1, \ldots, m\}: f^{j}(\widehat{y})<f^{j}(\bar{y})\right)\right)
$$

Analysis of Pareto efficiency allows decision-makers to exclude obviously inefficient objects from consideration, but it does not provide information about how much the objects which remain in consideration, are relatively effective. It is necessary to use techniques that allow to proportion the comparative significance of individual objects from the position of a holistic estimation of their efficiency. It means that we need to find an adequate way of comparing the individual characteristics of objects $s$ after which the object's comprehensive efficiency estimation $y \in Y$ is determined by purely mathematical way as $F(y) \equiv F_{s}(f(y)), y \in Y$. There are a number of established proportion methods in each subject field. In aviation, "fuel efficiency" and "weight efficiency", as well as several others are used as a complex criterion during aircraft's comparative estimation. The disadvantage of this approach is that the objective criteria are important, but they express only one property and are not universal. Therefore, the conclusions obtained with their use are questionable, since the use of other, to the same extend authoritative criterion, could lead to other conclusions.

The universal construction methods of criteria convolution are more reliable. The most famous of these is the linear convolution method, in which various characteris- 
tics are assigned numerical weight coefficients of relative importance. It is considered, that they can be obtained by averaging the opinion of many experts, involved by decision-makers for this purpose. Then

$$
\bar{F}(f)=\sum_{j=1}^{m} \alpha^{j} f^{j}, \alpha^{j} \geq 0, j=1, \ldots, m, \sum_{j=1}^{m} \alpha^{j}=1,
$$

where $\alpha^{j}, j=1, \ldots, m$ - weight coefficients.

The use of this method cannot be recommended during the design of such important objects as an aircraft for two main reasons.

\section{Confident judgments}

Let us notice, that the decision-maker made two judgments by choosing it:

- First of all, exactly this kind of account method for uncertainty in the form of linear convolution is fully adequate for this decision-making task,

- Secondly, exactly the chosen experts, the examination organization and methods of expert opinion processing load to absolutely reliable values of weight coefficients.

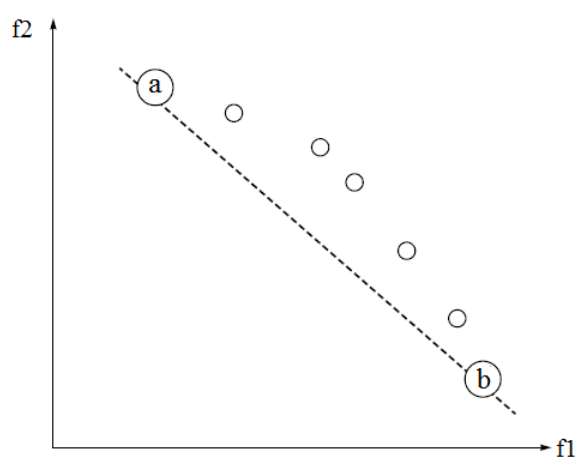

Fig. 1. Example of incorrect linear convolution

Both judgments can be challenged by reasonable positions. First of all, the linear convolution may not see some Pareto-optimal objects for any values of weight coefficients. For instance, on Figure 1 all objects for two minimized objects, images of which lie above the dotted line in a criterion space, will not be recognized as the most rational for any weight coefficient values in linear convolution, although they are Pareto-optimal objects [4]. Thus, this example shows that the use of the linear convolution penalizes a natural requirement for multiple comparison methods of individual object's characteristics $S$ : any Pareto-optimal variant from the set of admissible solutions must correspond to at least one function $F_{S}(f) \in S$, the use of which provide the most rational solution. If this requirement is failure to comply, it reduces the select possibilities of decision-makers by purely mathematical features of the aircraft, which is unacceptable. The subjectivity of weight coefficients' determination by means of expert examination is evident. In addition, the need to attract qualified experts when- 
ever the decision-maker wants to take a new look at the situation, greatly reduces the data analysis capabilities.

Actually, the decision-maker can reasonably make only two types of judgments.

The confident judgment of the first type. Decision-maker person (DMP) with his confidence may include various particular criteria to different group of importance. For example, "criteria 1 and 4 are the most important ones, criteria 2 and 6 are merely importance, and criterion 5 has the lowest importance". Let us note, that we do not assume that decision-maker provide a qualitative estimation of the relative importance degree for particular criteria, it refers only to the qualitative comparison which is optional.

The confident judgment of the second type. If desired, the decision-maker can construct the pairs of Pareto-incomparable vectors of particular criteria, for which he is certain that one of the vectors is better that another. It is not required that the vectors represent the efficiency of any real objects. If $f_{1}$ and $f_{2}-$ in which $f_{1}$ is surely better than $f_{2}$, it implements the following restriction on the set $S$ :

$$
S \subset\{s)\}: F_{s}\left(f_{1}\right) \leq F_{s}\left(f_{2}\right) \forall s \in S \text {. }
$$

Based only on these two types of judgments, the method which proportion particular characteristics into a single numerical object's characteristic, represented in the database, was developed in $[5,6]$. It is called confident judgments method.

\section{Stages of confident judgments' method}

Stage 1. The uncertainty profile is constructed for the solving problem. It shows the range of complex efficiency criterion values for this decision within all possible ways to take into account the uncertainty for each design solution. The uncertainty profile is given by a pair of functions, which are defined on the set: minorant $m(y)=$

$$
\min _{s \in S} F(f(y)) \text { and majorant } M(y)=\min _{s \in S} F(f(y)) \text {. }
$$

It should be noted, that obviously irrational decisions $z \in Y$, for which there are better solutions $\bar{z} \in Y$ by complex criterion in all possible ways of uncertainty, can be identified. The identify conditions for such solutions have the following form:

$$
\exists \bar{z} \in Y: M(\bar{z})<m(z) \text {. }
$$

The main purpose of uncertainty profile is to give decision-makers information about the impact of uncertainty during decision-making in the problem. Adding confident judgments, he will be able to estimate how they reduce the uncertainty.

Stage 2. If it is possible, the set of uncertainties narrows by taking into account the decision-maker's confident judgments. The uncertainty accounting methods, which do not correspond to this judgments, are eliminated when we are using confident judgments of the first type. When we have got the confident judgments of the second type, conditions (6) are added to the set description, which eliminates those uncertainty accounting methods that do not carry out these judgments.

At the end of first two stages the initial set of uncertainties can be narrowed. This affect the uncertainty profile of the problem, but it is unlikely to remain only a single element in it, or all variants except one will be eliminated from the plurality of solu- 
tions. Thus, the uncertainty retained in the problem. This will be a fatal uncertainty. All uncertainty account methods, which form this fatal uncertainty, are completely equal to the decision-maker as he has already use his ability to make additional content in the problem description using judgments of the first and second types. It is possible that the other types of confident judgments of decision-makers can be found, but they did not fundamentally change the situation: after their usage, fatal uncertainty will remain in the problem.

Stage 3. Rigid and soft ratings for solution variants are calculated, taking into account unavoidable uncertainty. In order not to introduce the unnecessary for understanding and application complex mathematical apparatus, we shall assume that the set contains a finite number of uncertainty accounting methods $S: S=\left\{S_{k}\right\}_{k=1, \ldots, K}$.

Then the rigid rating $R G(y)$ for $y \in Y$ solution is a fraction of uncertainty accounting method, in which the solution is the best compared to the other solutions:

$$
R G(y)=\frac{F_{k}(y) \leq F_{k}(z) \forall z \in Y}{K}, y \in Y
$$

(If any uncertainty accounting method has several best solutions, we should write $\frac{1}{p}$ instead of 1 in the numerator of rigid rating).

Soft Rating $R M(y)$ for $y \in Y$ decisions displays the average comprehensive efficiency if this solution compared with solutions, which are the best in different ways of uncertainty concideration:

$$
R M=\frac{\sum_{k=1}^{K} \frac{F_{s_{k}}(f(y))}{\max _{y \in Y} F_{s_{k}}(f(y))}}{K} .
$$

Stage 4. Decision-maker recognize that the possibility of further uncertainty reducing is exhausted due to its confident judgments. Finally, he chooses a solution with the best (lowest) rigid rating as the most efficient solution. If there are several solutions, we will choose the one, which has the best (lowest) soft rating, as the most efficient solution.

\section{Data analysis using confident judgments method}

Let us show the use of confident judgments method for data analysis of passenger aircrafts in terms of their operational characteristics. Table 2 shows a fragment of the database. 
Table 2. A fragment of database for passenger aircrafts

\begin{tabular}{|c|c|c|c|c|c|c|c|c|c|c|c|c|c|}
\hline 穿 & 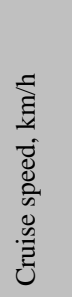 & 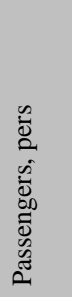 & 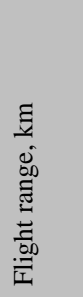 & 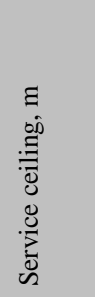 & 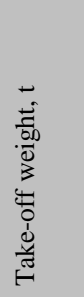 & 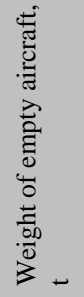 & 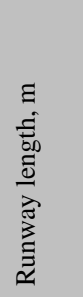 & 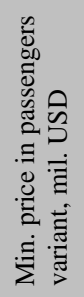 & 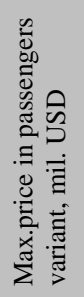 & 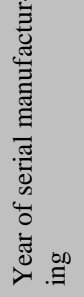 & 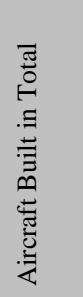 & 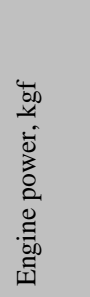 & 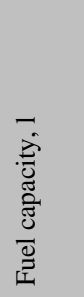 \\
\hline $\begin{array}{c}\text { Boeing } \\
737-200\end{array}$ & 905 & 120 & 2960 & 10670 & 49,40 & 27,17 & 1830 & 3,00 & 11,00 & 1967 & 3660 & 13160 & 10790 \\
\hline $\begin{array}{c}\text { Boeing } \\
\text { 737-200 } \\
\text { Advance }\end{array}$ & 905 & 120 & 3700 & 10670 & 58,00 & 31,90 & 1830 & 3,00 & 11,00 & 1984 & 865 & 15780 & 16250 \\
\hline $\begin{array}{c}\text { Boeing } \\
737-300\end{array}$ & 910 & 128 & 4670 & 10200 & 62,80 & 34,54 & 1940 & 10,50 & 44,00 & 1984 & 1102 & 19940 & 20105 \\
\hline $\begin{array}{c}\text { Boeing } \\
737-400\end{array}$ & 910 & 168 & 3870 & 11300 & 68,10 & 37,46 & 1920 & 18,50 & 48,00 & 1988 & 456 & 21340 & 23825 \\
\hline $\begin{array}{l}\text { Boeing } \\
737-500\end{array}$ & 910 & 108 & 5550 & 11300 & 60,55 & 33,30 & 1530 & 33,00 & 39,00 & 1990 & 385 & 18160 & 20105 \\
\hline $\begin{array}{c}\text { Boeing } \\
737-600\end{array}$ & 925 & 108 & 5910 & 12500 & 65,09 & 35,80 & 1880 & 32,00 & 39,00 & 1998 & 20 & 18160 & 26035 \\
\hline $\begin{array}{c}\text { Boeing } \\
737-700\end{array}$ & 925 & 128 & 5920 & 12500 & 69,40 & 38,17 & 2040 & 39,00 & 46,00 & 1997 & 15 & 21830 & 26035 \\
\hline $\begin{array}{l}\text { Boeing } \Gamma \\
737-800\end{array}$ & 925 & 189 & 5370 & 12500 & 78,24 & 43,03 & 2040 & 48,00 & 54,00 & 1998 & 20 & 23860 & 26035 \\
\hline Tu-204 & 850 & 210 & 3700 & 12600 & 94,60 & 52,03 & 1550 & 20,00 & 25,00 & 1994 & 15 & 32280 & 32000 \\
\hline $\begin{array}{c}\text { Tu-204- } \\
100\end{array}$ & 850 & 210 & 5200 & 12600 & $\begin{array}{c}103,0 \\
0\end{array}$ & 56,65 & 1750 & 22,00 & 27,00 & 1995 & 20 & 32280 & 32000 \\
\hline $\begin{array}{c}\text { Tu-204- } \\
120\end{array}$ & 850 & 210 & 5200 & 12100 & $\begin{array}{c}103,0 \\
0\end{array}$ & 56,65 & 1800 & 25,00 & 29,00 & 1997 & 26 & 39000 & 29900 \\
\hline $\begin{array}{l}\text { Tu-204- } \\
200\end{array}$ & 850 & 210 & 6200 & 12100 & $\begin{array}{c}110,7 \\
5\end{array}$ & 60,91 & 2050 & 30,00 & 35,00 & 1996 & 23 & 32280 & 32000 \\
\hline $\begin{array}{c}\text { Tu-204- } \\
300\end{array}$ & 850 & 210 & 3400 & 12600 & 86,00 & 47,30 & 2050 & 35,00 & 40,00 & 1997 & 22 & 32280 & 32000 \\
\hline $\begin{array}{c}\text { Airbus } \\
\text { Industry } \\
\text { A319-110 }\end{array}$ & 900 & 124 & 4910 & 11275 & 68,00 & 37,40 & 1750 & 35,00 & 35,00 & 1996 & 15 & 21340 & 23860 \\
\hline $\begin{array}{c}\text { Airbus } \\
\text { Industry } \\
\text { A321-200 }\end{array}$ & 900 & 185 & 5000 & 10676 & 89,00 & 48,95 & 2000 & 46,20 & 51,00 & 1996 & 1000 & 29960 & 23700 \\
\hline
\end{tabular}

Analyzing this data, first of all, we will use one on the traditional comprehensive performance criteria - fuel efficiency. In this case, the only Pareto-optimal object is Tu-204-200, which rigid rating is equal to $100 \%$ (Column 2, Table 3). Its fuel efficiency is equal to 19,6644 grams/pass*km, while the nearest Tu-204-120 it is 23.44 grams/pass*km. At the same time, we can use the other criteria - weight efficiency, which is calculated as the aircraft's ration of takeoff weight to the number of passengers. In this instance, the only Pareto-optimal variant with $100 \%$ rigid rating is anoth- 
er one object - Boeing Боинг 737-400 (Column 3 of Table 3), the weight efficiency of which is $0,41 \mathrm{t}$ /pass, whereas Tu-204-120 has 0,49 .

Table 3. The result of data analysis for passenger aircrafts

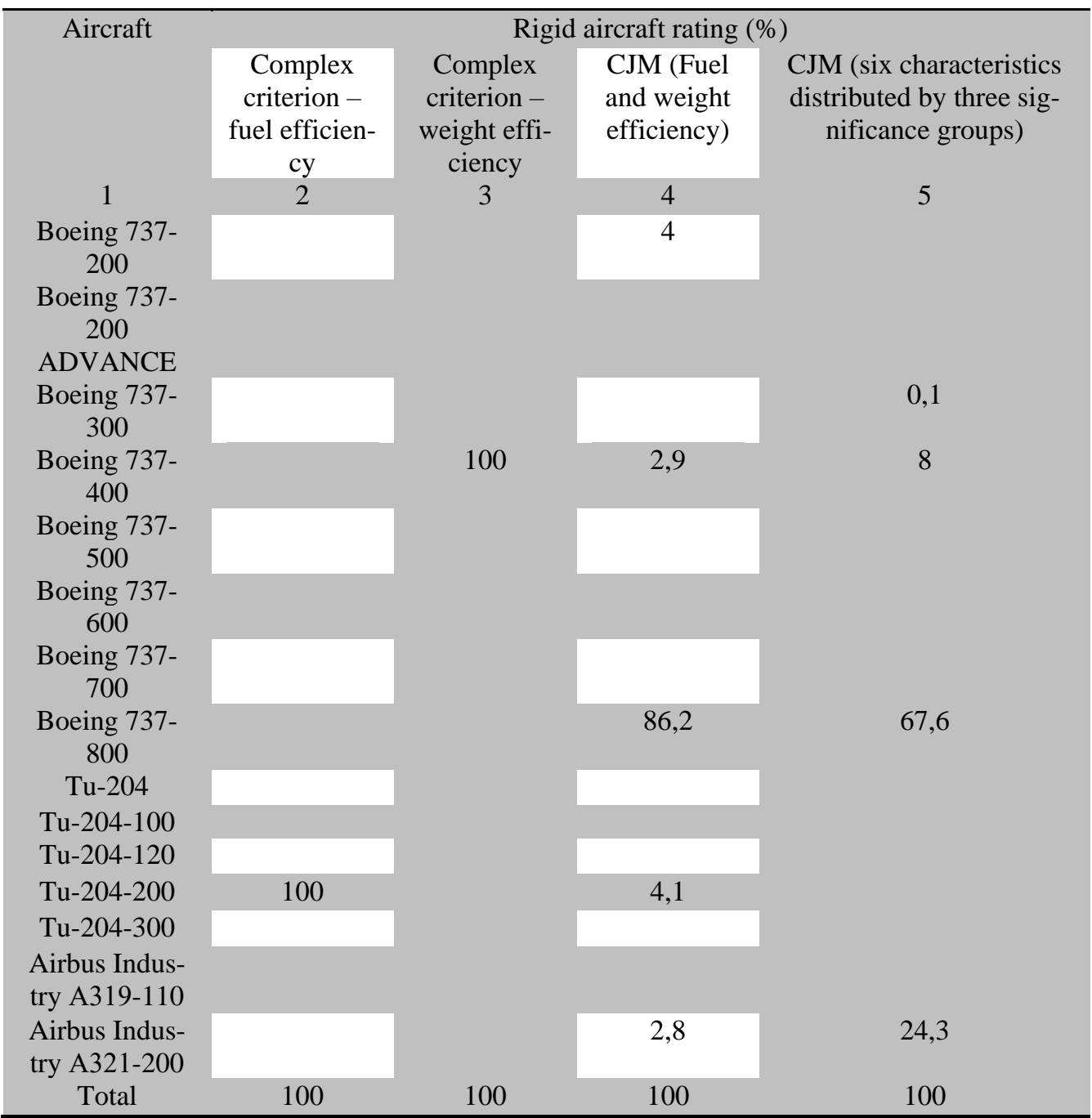

Let us use confident judgments method to analyze the data. If we suppose that the decision-maker wants to use both of complex criteria, which were mentioned above, without giving preferences to any of them in order to organize data, we will receive the results shown in column 4 of Table 3. In this case, fire aircrafts are worthy of consideration (Pareto-optimal): Boeing 737-800, Tu-204-200, Boeing 737-200, Boeing 737-400 and Airbus Industry A321-200, while their efficiency was compared in relative scale. According to this, Boeing737-800 leads by a wide margin - its rigid rating is equal to $86,2 \%$. Each of other listed aircrafts has only few percent of rigid rating. 
However, applying confident judgments method, there is no need to bring subjectivity in the data analysis, coupled with the use of traditional complex criteria. It is enough to list the primary characteristics which are significant to the maintenance viewpoint. They are:

- Cruise speed,

- Number of passengers,

- Range,

- Minimum price in passenger variant,

- Maximum price in passenger variant,

- Fuel capacity.

Cruise speed and number of passengers are the most significant criteria. Taking into account the variety of routes for various distances, on which aircrafts are operated within its capabilities, the range and fuel capacity are the following on the importance. They also influence on the running costs, as they are transferred to the minimum and maximum ticket price of an aircraft. Thus, the criteria are distributed into three groups of significance. The results are shown in column 5 of Table 3. Boeing 737-800 saves leading positions, and Airbus Industry A321-200 follows it with a considerable margin.

\section{Conclusion}

Thus, the article shows that the application of confident judgments method for analysis of large databases opens new flexible opportunities for its users.

\section{References}

1. Larichev O. Theory and methods for decision-methods. Logos, 2000; 295.

2. Larichev O. Verbal decision analysis. ISI RAS, Science, 2006; 181.

3. Smirnov O, Padalko S, Piyabskiy S. CAD: the formation and functioning of project modules. Mechanical engineering, 1987; 272.

4. Malyshev V, Piyavskiy B, Piyavsliy S. Decision-making methods taking into account the variety of uncertainty conditions. Izvestiya RAS, Theory and control systems, 2001; 1: 4661.

5. Piyavskiy S. Two new top-level concepts in the ontology of multi-criteria optimization. Designing ontology, 2013; 1(7): 65-68.

6. Malyshev V, Piyavskiy S. Confident judgments method for making multi-criteria decisions. RAS, Theory and control systems, 2015; 5: 90-101.

7. World passenger aircrafts. Argus, 1997; 336 